\title{
COMPLEX GEODESICS, THEIR BOUNDARY REGULARITY, AND A HARDY-LITTLEWOOD-TYPE LEMMA
}

\author{
Gautam Bharali \\ Indian Institute of Science, Department of Mathematics \\ Bangalore 560012, India; bharali@math.iisc.ernet.in
}

\begin{abstract}
We begin by giving an example of a smoothly bounded convex domain that has complex geodesics that do not extend continuously up to $\partial \mathbf{D}$. This example suggests that continuity at the boundary of the complex geodesics of a convex domain $\Omega \Subset \mathbf{C}^{n}, n \geq 2$, is affected by the extent to which $\partial \Omega$ curves or bends at each boundary point. We provide a sufficient condition to this effect (on $\mathcal{C}^{1}$-smoothly bounded convex domains), which admits domains having boundary points at which the boundary is infinitely flat. Along the way, we establish a Hardy-Littlewood-type lemma that might be of independent interest.
\end{abstract}

\section{Introduction}

Let $\Omega$ be a bounded domain in $\mathbf{C}^{n}$ and let $\mathbf{D}$ denote the open unit disc centered at $0 \in \mathbf{C}$. A holomorphic map $f: \mathbf{D} \rightarrow \Omega$ is called a complex geodesic of $\Omega$ if it is an isometry for the Kobayashi distances on $\mathbf{D}$ and $\Omega$ (since $\Omega$ is bounded, the Kobayashi pseudodistance on $\Omega$ is a true distance). These objects provide the primary motivation for this work. Along the way, we prove a result in one complex variable that arose from our need for a type of Hardy-Littlewood lemma on D. Since the latter topic is familiar to a large number of readers, we defer its discussion to Section 2.

A fundamental theorem about the existence of complex geodesics is the following result:

Result 1.1. (Lempert, [11]) Let $\Omega$ be a bounded strictly convex domain in $\mathbf{C}^{n}$ with $\mathcal{C}^{3}$-smooth boundary.

(a) Given any two distinct points $z_{1}, z_{2} \in \Omega$, there exists a complex geodesic of $\Omega$ whose image contains $z_{1}$ and $z_{2}$.

(b) If, furthermore, $\Omega$ is strongly convex, then every complex geodesic $f: \mathbf{D} \rightarrow \Omega$ extends to a map of class $\mathcal{C}^{1}(\overline{\mathbf{D}})$.

A domain $\Omega$ is said to be strictly convex if for any two points $z_{1}, z_{2} \in \bar{\Omega}$ the open segment $\left\{t z_{1}+(1-t) z_{2}: 0<t<1\right\} \subset \Omega$. The strongly convex domains form a proper subclass of the class of strictly convex domains: a convex domain $\Omega$ is said to be strongly convex if it has a $\mathcal{C}^{2}$-smooth boundary and the second fundamental form of $\partial \Omega$ is strictly positive definite.

The analogue of part (a) of Result 1.1 for all convex domains in general was established by Royden and Wong [13]. The Royden-Wong extension of Lempert's theorem does not, however, make any assertions about the boundary regularity of complex geodesics.

doi:10.5186/aasfm.2016.4116

2010 Mathematics Subject Classification: Primary 30H05, 32H40; Secondary 32F45.

Key words: Boundary regularity, complex geodesics, Hardy-Littlewood lemma.

This work is supported in part by a UGC Centre for Advanced Study grant. 
One cannot, in general, expect the complex geodesics of a convex domain to extend even continuously up to $\partial \mathbf{D}$. To see this, consider the polydisc $\mathbf{D}^{n}, n \geq 2$ : it is easy to see that $f=\left(f_{1}, \ldots, f_{n}\right): \mathbf{D} \rightarrow \mathbf{D}^{n}$ is a complex geodesic if and only if at least one of $f_{1}, \ldots, f_{n}$ is an automorphism of $\mathbf{D}$. By choosing any one map among $f_{1}, \ldots, f_{n}$ to be such that it does not extend continuously up to $\partial \mathbf{D}$, we see that complex geodesics do not, in general, extend continuously up to $\partial \mathbf{D}$. This example might lead one to suspect that the non-smoothness of $\partial \Omega$ is the chief reason that a complex geodesic does not extend continuously up to $\partial \mathbf{D}$. However, non-smoothness of $\partial \Omega$ is not the relevant issue, as the following example shows:

Example 1.2. An example of a bounded convex domain with $\mathcal{C}^{\infty}$-smooth boundary having complex geodesics that do not extend continuously to $\partial \mathbf{D}$.

Consider a complex geodesic of $\mathbf{D}^{2}, f=\left(f_{1}, f_{2}\right)$, where $f_{1}$ is an automorphism of $\mathbf{D}$ and $f_{2}$ is a bounded holomorphic function with $\left|f_{2}\right|<1 / 2$ that does not extend continuously up to $\partial \mathbf{D}$. Let $\Omega$ be any convex domain having $\mathcal{C}^{\infty}$-smooth boundary such that $\mathbf{D} \times D(0 ; 1 / 2) \subset \Omega \varsubsetneqq \mathbf{D}^{2} . K_{G}$ will denote the Kobayashi distance on the domain $G$ and p the Poincaré metric. Then:

$$
\mathrm{p}\left(\zeta_{1}, \zeta_{2}\right)=K_{\mathbf{D}^{2}}\left(f\left(\zeta_{1}\right), f\left(\zeta_{2}\right)\right) \leq K_{\Omega}\left(f\left(\zeta_{1}\right), f\left(\zeta_{2}\right)\right) \leq \mathrm{p}\left(\zeta_{1}, \zeta_{2}\right) \quad \forall \zeta_{1}, \zeta_{2} \in \mathbf{D} .
$$

The equality above encodes the fact that $f$ is a complex geodesic of $\mathbf{D}^{2}$ while the first inequality is the distance-decreasing effect of the inclusion that maps $\Omega \hookrightarrow \mathbf{D}^{2}$. Thus, $f$ is a complex geodesic of $\Omega$, but it does not extend continuously up to $\partial \mathbf{D}$.

This example suggests that the property of a convex domain $\Omega$ that affects the boundary behaviour of generic complex geodesics is the flatness of $\partial \Omega$ or the extent to which $\partial \Omega$ curves or bends at each boundary point. This notion is supported by part (b) of Result 1.1. (We emphasize the word "generic" here because even in domains with rough boundaries there may exist points $z_{1}$ and $z_{2}$ in special position, consider $\mathbf{D}^{2}$ for instance, such that some complex geodesic containing them extends continuously up to $\partial \mathbf{D}$. We will not address this type of non-generic phenomena in this work.) Our notion is further supported by a result of Mercer [12, Proposition 2.9], which states that all complex geodesics of a bounded $m$-convex domain - see Definition 2.7 in [12] — extend to maps that are Hölder-continuous on $\overline{\mathbf{D}}$ (where the Hölder exponent depends on the parameter $m$ ).

If a domain $\Omega$ is smoothly bounded and $m$-convex, then for each $w \in \partial \Omega$ the (complex) order of contact of the complex line $w+\mathbf{C} v$, for each $v \in H_{w}(\partial \Omega):=$ $T_{w}(\partial \Omega) \cap i T_{w}(\partial \Omega), v \neq 0$, with $\partial \Omega$ at $w$ is at most $m$. In contrast, we will show that all complex geodesics of $\Omega$ extend continuously up to $\partial \mathbf{D}$ even if there are points $w \in \partial \Omega$ at which $w+\mathbf{C} v$, for some $v \in H_{w}(\partial \Omega)$, osculates $\partial \Omega$ to infinite (complex) order, provided $\partial \Omega$ exhibits some degree of bending in the complex-tangential directions. To make this precise when $\partial \Omega$ is merely $\mathcal{C}^{1}$-smooth requires a little effort. To this end, we need the following definition. (Henceforth, $B^{d}(a ; r)$ will denote the Euclidean ball in $\mathbf{C}^{d}$ with centre $a$ and radius $r$.)

Definition 1.3. Let $\Omega$ be a bounded convex domain in $\mathbf{C}^{n}, n \geq 2$, with $\mathcal{C}^{1}$ smooth boundary. Let $F: B^{n-1}(0 ; r) \rightarrow \mathbf{R}$ be a smooth function with $F(0)=0$ and $D F(0)=0$. We say that $F$ supports $\Omega$ from the outside if there exist constants $R_{0} \in$ $(0, r)$ and $s_{0}>0$ such that, for each $w \in \partial \Omega$, there exists a unitary transformation $\mathrm{U}_{w}$ satisfying:

- $\mathrm{U}_{w}\left(H_{w}(\partial \Omega)\right)=\left\{v \in \mathbf{C}^{n}: v_{n}=0\right\}$, and 
- $\mathrm{U}_{w}\left(\nu_{w}\right)=(0, \ldots, 0, i)$, where $\nu_{w}$ denotes the inward unit normal vector to $\partial \Omega$ at $w$,

such that, denoting the $\mathbf{C}$-affine map $v \longmapsto \mathbf{U}_{w}(v-w)$ as $\mathbf{U}^{w}$, we have

$$
\begin{aligned}
& \mathrm{U}^{w}(\bar{\Omega}) \cap B^{n-1}\left(0 ; R_{0}\right) \times\left(\left(-s_{0}, s_{0}\right)+i\left(-s_{0}, s_{0}\right)\right) \\
& \subset\left\{z=\left(z^{\prime}, z_{n}\right) \in B^{n-1}\left(0 ; R_{0}\right) \times\left(\left(-s_{0}, s_{0}\right)+i\left(-s_{0}, s_{0}\right)\right): \operatorname{Im}\left(z_{n}\right) \geq F\left(z^{\prime}\right)\right\} .
\end{aligned}
$$

Perhaps the most familiar examples of functions on $[0, \infty)$ that vanish to infinite order at 0 are the functions $\Psi_{\alpha}, \alpha>0$, defined as follows:

$$
\Psi_{\alpha}(x):= \begin{cases}e^{-1 / x^{\alpha}}, & \text { if } x>0 \\ 0, & \text { if } x=0\end{cases}
$$

These functions help us to translate the qualitative notion expressed prior to Definition 1.3 to give us the following result:

Theorem 1.4. Let $\Omega$ be a bounded convex domain in $\mathbf{C}^{n}, n \geq 2$, with $\mathcal{C}^{1}$-smooth boundary. Assume that $\Omega$ is supported from the outside by $F\left(z^{\prime}\right):=C \Psi_{\alpha}\left(\left\|z^{\prime}\right\|^{\alpha}\right)$ (writing $z=\left(z^{\prime}, z_{n}\right)$ for each $\left.z \in \mathbf{C}^{n}\right)$ for some $C>0$ and some $\alpha \in(0,1)$. Then, every complex geodesic of $\Omega$ extends continuously up to $\partial \mathbf{D}$.

Remark 1.5. Note that a domain that satisfies the hypothesis of Theorem 1.4 need not be strictly convex. For such a domain $\Omega$, it is possible for $\partial \Omega$ to contain line segments that point along the complex-normal direction at each of the boundary points through which they pass.

Remark 1.6. The condition in Theorem 1.4 might seem at first a bit artificial. However, readers familiar with the analysis of the boundary geometry of a domain around points that are not of finite type know that there exists no classification of the local normal forms for $\partial \Omega$, at infinite-type points in $\partial \Omega$, analogous to even the very general notion due to Catlin [1]. When $\partial \Omega$ contains points at which it is infinitely flat and yet $\partial \Omega$ is assumed to have low regularity globally, a way to model $\partial \Omega$ is through objects such as the one in Definition 1.3. Even then, choices need to be made. We have chosen the functions in (1.1) to underlie the functions supporting $\Omega$ because these are the most well-known functions that vanish to infinite order.

What can one say if the convex domain given is supported from the outside by $C \Psi_{\alpha}(\|\cdot\|)$ and $\alpha \geq 1$ ? This is a subtle question, but see Remark 1.7 below. In working with the latter functions, $\alpha=1$ usually marks a transition-point for methods that work well for $0<\alpha<1$. For illustrations of this, see, for instance, [4, Section 3], [6, Section 3] or [5].

The key quantitative ingredient in the proof of Theorem 1.4 is a simple extension of one of the Hardy-Littlewood lemmas to holomorphic functions on $\mathbf{D}$ whose derivatives have rather rapid growth. This extension leads to a characterization (which is unrelated to Theorem 1.4, but may be of independent interest) of a class of holomorphic functions that is strictly larger than every class of holomorphic functions on $\mathbf{D}$ having a Hölder-continuous extension to $\partial \mathbf{D}$. We discuss these matters in the next section. The proof of Theorem 1.4 will be given in Section 3 .

Remark 1.7. A few months after this paper was written, Zimmer showed, among other things, that if $\Omega$ is a $\mathbf{C}$-strictly convex domain having $\mathcal{C}^{1, \alpha}$-smooth boundary, then every complex geodesic of $\Omega$ extends continuously up to $\partial \mathbf{D}[14$, 
Corollary 1.8]. The domains in our Theorem 1.4 are, in Zimmer's terminology, Cstrictly convex, although [14, Corollary 1.8] addresses only those domains in Theorem 1.4 that have $\mathcal{C}^{1, \alpha}$-smooth boundaries. However, a $\mathbf{C}$-strictly convex $\Omega$ admits points $p \in \partial \Omega$ at which $\partial \Omega$ can be flat to any extent without containing a germ of a complex line at $p$. Zimmer's proof uses ingredients very different from those alluded to above. The constraint that $\partial \Omega$ be $\mathcal{C}^{1, \alpha}$-smooth arises from one of those ingredients.

\section{A Hardy-Littlewood-type lemma}

The phrase "Hardy-Littlewood-type lemma" refers in the present context to any type of result that, given a function $f \in \mathcal{O}(\mathbf{D})$ :

(a) tells us, based on the growth of $\left|f^{\prime}(\zeta)\right|$ in terms of $\operatorname{dist}(\zeta, \partial \mathbf{D})$, whether $f$ extends continuously up to $\partial \mathbf{D}$, and

(b) if possible, characterizes the extension of $f$ to $\partial \mathbf{D}$ in terms of its modulus of continuity.

We shall present here a result of this type. Our result arises from the following proposition, which is central to proving Theorem 1.4. Some notation: given an interval $E \subset \mathbf{R}, \mathbf{L}^{1}(E)$ will denote the $\mathbf{L}^{1}$-class with respect to the Lebesgue measure on $E$.

Proposition 2.1. Let $\Phi:\left[0, r_{0}\right) \rightarrow[0,+\infty]$ be a function of class $\mathbf{L}^{1}\left(\left[0, r_{0}\right)\right)$ for some $r_{0} \in(0,1)$. Let $f \in \mathcal{O}(\mathbf{D})$ and assume that

$$
\left|f^{\prime}\left(r e^{i \theta}\right)\right| \leq \Phi(1-r) \quad \forall r: 1-r_{0}<r<1 \text { and } \forall \theta \in \mathbf{R} .
$$

Then, $f$ extends continuously to $\partial \mathbf{D}$.

The proof of this statement is simple, and we shall skip some elementary details. The condition on $f^{\prime}$ implies, owing to the dominated convergence theorem, that the limit

$$
f^{\bullet}\left(e^{i \theta}\right):=\lim _{r \rightarrow 1^{-}} f\left(r e^{i \theta}\right)=f(0)+\lim _{r \rightarrow 1^{-}} e^{i \theta} \int_{0}^{r} f^{\prime}\left(s e^{i \theta}\right) d s
$$

exists for each $\theta \in \mathbf{R}$, and that this limit is uniform in $\theta$. This, together with the fact that, for a fixed $R \in(0,1)$, we can make $\left|f\left(R e^{i \theta_{1}}\right)-f\left(R e^{i \theta_{2}}\right)\right|$ as small as we wish by taking $\theta_{1}$ and $\theta_{2}$ sufficiently close to each other, implies that $f^{\bullet} \in \mathcal{C}(\partial \mathbf{D})$. The usual Poisson-integral argument establishes that $f^{\bullet}\left(e^{i \theta}\right)$ is the continuous extension of $f$ to $e^{i \theta} \in \partial \mathbf{D}$.

To proceed further, we must recall a definition. Given a function $g: \partial \mathbf{D} \longrightarrow \mathbf{C}$, we define the modulus of continuity of $g$ by

$$
\omega_{g}(\delta):=\sup _{|\theta-\phi| \leq \delta}\left|g\left(e^{i \theta}\right)-g\left(e^{i \phi}\right)\right|, \quad 0 \leq \delta \leq \pi .
$$

This concept can be defined in a much more general setting, but we shall restrict ourselves to $\mathbf{C}$-valued functions on $\partial \mathbf{D}$. Clearly

$$
g \in \mathcal{C}(\partial \mathbf{D} ; \mathbf{C}) \Longleftrightarrow \lim _{\delta \rightarrow 0^{+}} \omega_{g}(\delta)=0 .
$$

The classical Hardy-Littlewood lemma characterizes, in terms of the growth of $f^{\prime}$, functions $f \in \mathcal{O}(\mathbf{D})$ that extend continuously up to $\partial \mathbf{D}$ such that $\omega_{f} \bullet(\delta)=C \delta^{\alpha}$, $C>0$ and $0<\alpha \leq 1$, i.e., such that the boundary-value of $f$ belongs to a Hölder class on $\partial \mathbf{D}$. In this section, given $f \in \mathcal{O}(\mathbf{D}), f^{\bullet}$ will denote the radial boundary-value of $f$ (whenever it exists). 
Since very little is assumed about the function $\Phi$ in Proposition 2.1, characterizing the boundary-values $f^{\bullet}$ of the functions $f$ mentioned therein is probably not tractable. But it does raise a natural question: is there such a characterization of a class that:

1) includes, for instance, functions for which $\omega_{f} \bullet(\delta)=(\log (1 / \delta))^{-1}$ for $\delta$ close to 0 ; or

2) at least includes the case $\omega_{f} \bullet(\delta)=e^{-\alpha(\log (1 / \delta))^{1-\varepsilon}}, 0<\varepsilon<1$, for $\delta$ close to 0 ?

Observe: when $\varepsilon$ is close to 0 and $0<\alpha<1$, the values of the latter function are very close to $\delta^{\alpha}$ for $\delta \leq 1$, unless $\delta$ is extremely small. Yet, if there are any functions $f \in \mathcal{O}(\mathbf{D})$ for which (2) is true, then $f^{\bullet}$ would be rougher than any Hölder-continuous function.

In the recent literature, we have been introduced to classes of functions - defined by their moduli of continuity - that are not as rough as the boundary-values given by Proposition 2.1 but are considerably less regular than the Hölder-continuous functions: see, for instance, [3] by Dyakonov, or [10] by Kuusi-Mingione. The next definition describes a continuity class in the style of the latter paper, but which is large enough to include functions having the modulus of continuity described in (2).

Definition 2.2. Let $g \in \mathcal{C}(\partial \mathbf{D} ; \mathbf{C})$. We say that $g$ is $\log$-Dini continuous if $\omega_{g}$, the modulus of continuity of $g$, satisfies

$$
\int_{0}^{1}(\log (1 / x))^{n} \frac{\omega_{g}(x)}{x} d x<\infty \text { for } n=0,1,2, \ldots
$$

We are now in a position to state the main theorem of this section.

Theorem 2.3. Let $f \in \mathcal{O}(\mathbf{D})$. The function $f$ extends to a continuous function on $\overline{\mathbf{D}}$ such that $f\left(e^{i \cdot}\right)$ is $\log$-Dini continuous if and only if there exists a positive non-increasing function $\Phi:\left[0, r_{0}\right) \longrightarrow[0,+\infty]$, for some $r_{0} \in(0,1)$, such that:

(a) $(\log (1 / \cdot))^{n} \Phi$ is of class $\mathbf{L}^{1}\left(\left[0, r_{0}\right)\right)$ for each $n \in \mathbf{N}$; and

(b) $\left|f^{\prime}\left(r e^{i \theta}\right)\right| \leq \Phi(1-r)$ for all $r \in\left(1-r_{0}, 1\right)$ and for every $\theta \in \mathbf{R}$.

Furthermore, whenever this happens, $\omega_{f} \bullet(\delta)$ is dominated by $3\left(\int_{0}^{\delta} \Phi(x) d x\right)$ for $0 \leq$ $\delta<r_{0}$.

Proof. We first assume the existence of a $\Phi$ for which $(a)$ and $(b)$ hold true. From the paragraph following the statement of Proposition 2.1, we have that $f$ extends to a continuous function on $\partial \mathbf{D}$. We shall continue to denote this extension by $f$ and set $f^{\bullet}:=\left.f\right|_{\partial \mathbf{D}}$. We shall now deduce some information about $\omega_{f} \bullet$.

To this end, fix $\theta_{1}, \theta_{2} \in \mathbf{R}$ such that, for the moment, $0<\theta_{2}-\theta_{1}<\pi$. Let $\gamma^{r, \rho}:[0,1] \rightarrow \mathbf{D}$ be a path from $r e^{i \theta_{1}}$ to $r e^{i \theta_{2}}$ whose image comprises the radial segments $\left[r e^{i \theta_{1}}, \rho e^{i \theta_{1}}\right]$ and $\left[\rho e^{i \theta_{2}}, r e^{i \theta_{2}}\right]$, where $1-r_{0}<\rho<r<1$, and the (shorter) arc of the circle $\{z \in \mathbf{C}:|z|=\rho\}$ from $\rho e^{i \theta_{1}}$ to $\rho e^{i \theta_{2}}$. Owing to the holomorphicity of $f^{\prime}$, and the existence of the limits in (2.1), we have

$$
f\left(e^{i \theta_{2}}\right)-f\left(e^{i \theta_{1}}\right)=\lim _{r \rightarrow 1^{-}} \int_{\gamma^{r}, \rho} f^{\prime}(z) d z
$$

for any $\rho$ as above. (A point about notation: each integral below of the form $\int_{a}^{b}, a<$ $b \in \mathbf{R}$, that involves a non-negative function denotes the standard (hence unoriented) Lebesgue integral on the interval $[a, b]$.) Thus 


$$
\left|f\left(e^{i \theta_{2}}\right)-f\left(e^{i \theta_{1}}\right)\right| \leq 2 \int_{\rho}^{1} \Phi(1-s) d s+\int_{\theta_{1}}^{\theta_{2}} \Phi(1-\rho) d \theta .
$$

Let us write $I(x):=\int_{0}^{x} \Phi(s) d s$. From the last inequality, we can see that our temporary restriction on $\left(\theta_{1}, \theta_{2}\right)$ does not matter, and we have

$$
\begin{aligned}
\left|f\left(e^{i \theta_{2}}\right)-f\left(e^{i \theta_{1}}\right)\right| & \leq 2 I(1-\rho)+\left|\theta_{2}-\theta_{1}\right| \Phi(1-\rho) \\
& \leq\left(2+\frac{\left|\theta_{2}-\theta_{1}\right|}{1-\rho}\right) I(1-\rho) \quad \forall \theta_{1}, \theta_{2}:\left|\theta_{2}-\theta_{1}\right| \leq \pi .
\end{aligned}
$$

The second inequality follows from the fact that $\Phi$ is non-increasing. Let us define

$$
\varpi(\delta):= \begin{cases}3 I(\delta), & \text { if } 0 \leq \delta<r_{0}, \\ 2 \sup \left|f^{\bullet}\right|, & \text { if } r_{0} \leq \delta \leq \pi\end{cases}
$$

We shall make a choice for the free parameter $\rho$ in (2.4) based on $\left(\theta_{1}, \theta_{2}\right)$. Taking $\rho=1-\left|\theta_{2}-\theta_{1}\right|$ whenever $0<\left|\theta_{2}-\theta_{1}\right|<r_{0}$, we see that

$$
\left|f\left(e^{i \theta_{2}}\right)-f\left(e^{i \theta_{1}}\right)\right| \leq \varpi\left(\left|\theta_{2}-\theta_{1}\right|\right) \quad \forall \theta_{1}, \theta_{2}:\left|\theta_{2}-\theta_{1}\right| \leq \pi .
$$

It is evident from (2.5) that $\varpi$ is a majorant of $\omega_{f} \bullet$

From this last statement, it follows that to establish that $f^{\bullet}$ is $\log$-Dini continuous, it suffices to establish the inequality in (2.3) with

- $\omega_{g}$ replaced by $\varpi$, and

- the integral over the interval $[0,1]$ replaced by an integral over the interval $[0, R]$ for some small $R>0$ (since the integrand in (2.3) is unbounded only as $x \rightarrow 0^{+}$).

Let us fix some $R$ such that $0<R<r_{0}$. By Tonelli's theorem, we have:

$$
\begin{aligned}
\frac{1}{3} \int_{0}^{R}(\log (1 / x))^{n} \frac{\varpi(x)}{x} d x & =\int_{0}^{R} \int_{0}^{x}(\log (1 / x))^{n} \frac{\Phi(s)}{x} d s d x \\
& =\int_{0}^{R}\left[\int_{s}^{R} \frac{(\log (1 / x))^{n}}{x} d x\right] \Phi(s) d s \\
& =\frac{1}{n+1} \int_{0}^{R}\left[(\log (1 / s))^{n+1}-(\log (1 / R))^{n+1}\right] \Phi(s) d s<\infty
\end{aligned}
$$

for each $n \in \mathbf{N}$. The last assertion is just the condition $(a)$. From our remarks above, it follows that $f^{\bullet}$ is $\log$-Dini continuous.

Conversely, let us assume that $f$ extends to a continuous function on $\overline{\mathbf{D}}$ and that $f^{\bullet}$ is $\log$-Dini continuous. Then, for any $r e^{i \phi} \in \mathbf{D}$, taking $\phi \in(-\pi, \pi]$, the Cauchy integral theorem gives us

$$
f^{\prime}\left(r e^{i \phi}\right)=\frac{1}{2 \pi} \int_{-\pi}^{\pi} \frac{f\left(e^{i \theta}\right)}{\left(e^{i \theta}-r e^{i \phi}\right)^{2}} e^{i \theta} d \theta=\frac{1}{2 \pi} \int_{-\pi}^{\pi} \frac{f\left(e^{i \theta}\right)-f\left(e^{i \phi}\right)}{\left(e^{i \theta}-r e^{i \phi}\right)^{2}} e^{i \theta} d \theta .
$$

Setting $\tau:=\theta-\phi$, whence $\left|f\left(e^{i \theta}\right)-f\left(e^{i \phi}\right)\right|$ transforms to $\left|f\left(e^{i(\phi+\tau)}\right)-f\left(e^{i \phi}\right)\right|$, we have

$$
\left|f^{\prime}\left(r e^{i \phi}\right)\right| \leq \frac{1}{\pi} \int_{0}^{\pi} \frac{\omega_{f} \bullet(\tau)}{r^{2}-2 r \cos \tau+1} d \tau \equiv \Phi(1-r) .
$$

We would be done if we could find some small $R>0$ such that $\int_{0}^{R}(\log (1 / x))^{n} \Phi(x) d x<$ $\infty$ for each $n \in \mathbf{N}$. 
Note that

$$
\begin{aligned}
r^{2}-2 r \cos \tau+1 & =(1-r)^{2}+4 r \sin ^{2}(\tau / 2) \geq(1-r)^{2}+4 r(\tau / \pi)^{2} \\
& \geq(1-r)^{2}+(\tau / \pi)^{2} \quad \forall r \in(1 / 4,1), \forall \tau \in[0, \pi] .
\end{aligned}
$$

Thus, if we fix $R \in(0,3 / 4)$ and set $x:=(1-r)$, then it suffices to establish the convergence of the following integrals:

$$
I_{n}:=\frac{1}{\pi} \int_{0}^{R}(\log (1 / x))^{n}\left[\int_{0}^{\pi} \frac{\omega_{f} \bullet(\tau)}{x^{2}+(\tau / \pi)^{2}} d \tau\right] d x, \quad n \in \mathbf{N} .
$$

Fix a $\delta \in(0, R)$ and let $I_{n}(\delta)$ denote the integral over $[\delta, R]$ of the integrand on the right-hand side above. Making the change of variable $y:=\tau / \pi x$ in the inner integral, we get

$$
\begin{aligned}
I_{n}(\delta) & =\int_{\delta}^{R} \frac{(\log (1 / x))^{n}}{x}\left[\int_{0}^{1 / x} \frac{\omega_{f} \cdot(\pi x y)}{1+y^{2}} d y\right] d x \\
& \leq \int_{\delta}^{R}(\log (1 / x))^{n} \frac{\omega_{f} \bullet(x)}{x}\left[\int_{0}^{1 / x} \frac{1+\pi y}{1+y^{2}} d y\right] d x \\
& \leq C \int_{\delta}^{R}(\log (1 / x))^{n} \frac{\omega_{f} \bullet(x)}{x}[1+\log (1 / x)] d x
\end{aligned}
$$

where $C>0$ is a constant that does not depend on $x, n$ or $\delta$. The first inequality above follows from the standard inequality $\omega_{f} \bullet(\lambda x) \leq(\lambda+1) \omega_{f} \bullet(x)$ for all $\lambda \geq 0$ (and sufficiently small that $\omega_{f} \bullet(\lambda x)$ makes sense); see $[2$, Chapter $2, \S 6]$. Since $f^{\bullet}$ is $\log$-Dini continuous, the integrands in $(2.6)$ are, in fact, of class $\mathbf{L}^{1}([0, R])$ for each $n \in \mathbf{N}$. Thus, it follows from the above estimate that $I_{n}<\infty$ for each $n \in \mathbf{N}$.

The final assertion of the theorem has already been established in the argument leading from the inequality (2.4) to the inequality (2.5).

One may ask whether there are any functions $f$ in the class $\mathcal{O}(\mathbf{D}) \cap \mathcal{C}(\overline{\mathbf{D}})$ beyond those already described by the classical Hardy-Littlewood lemma for which $\omega_{f} \bullet$ is as in the above theorem. We address this question by the following:

Example 2.4. There exist functions $f \in \mathcal{O}(\mathbf{D}) \cap \mathcal{C}(\overline{\mathbf{D}})$ such that $f^{\bullet}$ is $\log$-Dini continuous but belongs to no Hölder class.

Pick a function $\psi \in \mathcal{C}(\partial \mathbf{D} ; \mathbf{R})$ such that

$$
\omega_{\psi}(\delta)=\exp \left(-C(\log (1 / \delta))^{1-\varepsilon}\right) \quad \text { for } \delta \text { close to } 0,
$$

where $C>0$ and $0<\varepsilon<1$, and $\psi$ is not in any Hölder class. It is an elementary exercise to show that

$$
\int_{0}^{1}(\log (1 / x))^{n} \frac{e^{-C(\log (1 / x))^{1-\varepsilon}}}{x} d x<\infty \text { for } n=0,1,2, \ldots
$$

Let $\widetilde{\psi}$ denote the conjugate function of $\psi$. Then, by the Privalov-Zygmund estimate, there exists a constant $K>0$ such that

$$
\omega_{\widetilde{\psi}}(\delta) \leq K\left[\int_{0}^{\delta} \frac{\omega_{\psi}(x)}{x} d x+\delta \int_{\delta}^{\pi} \frac{\omega_{\psi}(x)}{x^{2}} d x\right] .
$$

This calls for a somewhat careful estimation of the two integrals above. First, by decomposing $(0, \delta]$ as $(0, \delta]=\cup_{j=0}^{\infty}\left[2^{-(j+1)} \delta, 2^{-j} \delta\right]$ (by $(2.7)$, the integral of $\omega_{\psi}(x) / x$ 
over $(0, \delta]$ is the same as the integral over $[0, \delta])$, we have

$$
\begin{aligned}
\int_{0}^{\delta} \frac{e^{-C(\log (1 / x))^{1-\varepsilon}}}{x} d x & \leq \sum_{j=0}^{\infty} \exp \left\{-C\left(\log \left(\frac{2^{j}}{\delta}\right)\right)^{1-\varepsilon}\right\} \\
& \leq \sum_{j=0}^{\infty} \exp \left[-2^{-\varepsilon} C\left((j \log 2)^{1-\varepsilon}+(\log (1 / \delta))^{1-\varepsilon}\right)\right] \\
& =K^{\prime} \exp \left(-2^{-\varepsilon} C\left(\log (1 / \delta)^{1-\varepsilon}\right) \quad \text { for } \delta \text { close to } 0\right.
\end{aligned}
$$

The second inequality above arises from the fact that, close to $0, \omega_{\psi}$ is a concave function. Let $R>0$ be such that $\omega_{\psi}$ is concave on $[0, R]$. Then, since $\omega_{\psi}(0)=0$, $x \longmapsto \omega_{\psi}(x) / x$ is a decreasing function on $[0, R]$. Using this fact, we get the crude, but adequate, estimate

$$
\delta \int_{\delta}^{\pi} \frac{e^{-C(\log (1 / x))^{1-\varepsilon}}}{x^{2}} d x \leq \log \left(\frac{R}{\delta}\right) e^{-C(\log (1 / \delta))^{1-\varepsilon}}+O(\delta) .
$$

It is easy to see directly from the estimate $(2.8)$ that $\lim _{\delta \rightarrow 0^{+}} \omega_{\widetilde{\psi}}(\delta)=0$. Thus, we conclude from $(2.2)$ that $\widetilde{\psi} \in \mathcal{C}(\partial \mathbf{D} ; \mathbf{R})$. Then, by definition, $(\psi+i \widetilde{\psi})$ is the boundary value of a function $\Psi \in \mathcal{O}(\mathbf{D}) \cap \mathcal{C}(\overline{\mathbf{D}})$. Now (2.7), taken together with the estimates (2.9) and (2.10), implies that $\Psi^{\bullet}$ is log-Dini continuous. However, since $\psi=(\operatorname{Re} \Psi)^{\bullet}$ was chosen so that it does not belong to any Hölder class, $\Psi^{\bullet}$ is not in any Hölder class either.

Remark 2.5. With more intensive analysis, one can show that the holomorphic functions constructed in Example 2.4 are such that the modulus of continuity of $f^{\bullet}$ is $O\left(\omega_{\psi}\right)$. That $(2.9)$ can be improved is not hard to see: one uses a better lower bound for $\left(\log \left(2^{j}\right)+\log (1 / \delta)\right)^{1-\varepsilon}$ in the step preceding $(2.9)$. The estimate $(2.10)$ is rather crude. It can be improved as desired, but this requires some effort. Since this is not the main thrust of the present section, we shall not elaborate any further on the last point.

\section{The proof of Theorem 1.4}

We now return to several complex variables. We begin by presenting some notation. If $\Omega$ is a bounded domain in $\mathbf{C}^{n}, z \in \Omega$ and $v \in \mathbf{C}^{n} \backslash\{0\}$, then

$$
\begin{aligned}
d_{\Omega}(z):= & \text { the Euclidean distance of } z \text { from } \partial \Omega, \\
r_{\Omega}(z ; v):= & \text { the radius of the largest complex-affine closed disc, centered at } z \\
& \text { and tangent to } v, \text { that is contained in } \bar{\Omega} .
\end{aligned}
$$

The crux of the proof of Theorem 1.4 is to find an estimate for $f^{\prime}$, where $f: \mathbf{D} \rightarrow$ $\Omega$ is a complex geodesic. One could then try to apply Proposition 2.1 to deduce continuous extension to $\partial \mathbf{D}$. If we have a reasonably good estimate for the Kobayashi pseudometric on $\Omega$, where $\Omega$ is as in Theorem 1.4, then we can use it to estimate $f^{\prime}$. This explains the need for the following result of Graham:

Result 3.1. (Graham, [8]) Let $\Omega$ be a bounded convex domain in $\mathbf{C}^{n}$ and let $\kappa_{\Omega}(z ; \cdot)$ denote the Kobayashi metric on $\Omega$ at the point $z \in \Omega$. Then:

$$
\frac{\|v\|}{2 r_{\Omega}(z ; v)} \leq \kappa_{\Omega}(z ; v) \leq \frac{\|v\|}{r_{\Omega}(z ; v)} \quad \forall z \in \Omega \text { and } \forall v \in \mathbf{C}^{n} \text {. }
$$


We must point out that in this section all distances and norms on $\mathbf{C}^{n}$ will be the Euclidean distance and the Euclidean norm, both denoted by $\|\cdot\|$.

To carry out the programme sketched above, we will require explicit estimates on $r_{\Omega}$. This is the role of the following lemma:

Lemma 3.2. Let $\Omega$ be as in Theorem 1.4. There exists a compact subset $K$ of $\Omega$ such that for each $z \in \Omega \backslash K$,

$$
r_{\Omega}(z ; v) \leq 2\left[\log \left(\frac{C}{d_{\Omega}(z)}\right)\right]^{-1 / \alpha} \quad \forall v \in \mathbf{C}^{n} \backslash\{0\},
$$

where $C$ and $\alpha$ are the constants appearing in Theorem 1.4.

Proof. Define $x_{0}$ as follows (it is not hard to argue that the set on the right is finite):

$$
x_{0}:=\min \left[\{C / 2\} \cup\left\{x \in(0, C): x=[\log (C / x)]^{-1 / \alpha}\right\}\right] .
$$

Let $s_{0}$ and $R_{0}$ be as given by Definition 1.3 with $F=C \Psi_{\alpha}(\|\cdot\|)$, and write

$$
\mathbf{M}:=\left\{z=\left(z^{\prime}, z_{n}\right) \in \mathbf{C}^{n}: \operatorname{Im}\left(z_{n}\right)=C \Psi_{\alpha}\left(\left\|z^{\prime}\right\|\right)\right\} .
$$

We can find a compact subset $K$ of $\Omega$ such that whenever $z \in \Omega \backslash K$,

- $d_{\Omega}(z)<\min \left(s_{0}, x_{0}\right)$; and

- For any point $w(z) \in \partial \Omega$ that satisfies $d_{\Omega}(z)=\|z-w(z)\|$, every complex line of the form

$$
\mathrm{U}^{w(z)}(z+\mathbf{C} v)\left(=\left(0, \ldots, 0, i \cdot d_{\Omega}(z)\right)+\mathbf{C U}_{w(z)}(v)\right), v \in H_{w(z)}(\partial \Omega) \backslash\{0\},
$$

intersects $\mathbf{M}$ in a circle of radius $\left[\log \left(C / d_{\Omega}(z)\right)\right]^{-1 / \alpha}=\left(C \Psi_{\alpha}\right)^{-1}\left(d_{\Omega}(z)\right)$.

Here $\mathbf{U}^{w(z)}$ is as described in Definition 1.3. For each $z \in \Omega \backslash K$, let us fix a $w(z)$ for the remainder of this proof. The above implies that if $z \in \Omega \backslash K$, then

$$
r_{\Omega}(z ; v) \leq\left[\log \left(\frac{C}{d_{\Omega}(z)}\right)\right]^{-1 / \alpha} \quad \forall v \in H_{w(z)}(\partial \Omega) \backslash\{0\} .
$$

Of course, for all such $z$, we also have

$$
r_{\Omega}(z ; v)=d_{\Omega}(z) \quad \forall v \in H_{w(z)}(\partial \Omega)^{\perp} \backslash\{0\} .
$$

(The orthogonal complement here is with respect to the standard Hermitian inner product on $\mathbf{C}^{n}$.)

Fix a $z \in \Omega \backslash K$, consider a general unit vector $v \in \mathbf{C}^{n} \backslash\{0\}$, and let $\theta_{v} \in \mathbf{R}$ be such that

$$
e^{i \theta_{v}} \mathbf{U}_{w(z)} v=\left(e^{i \theta_{v}}\left(\mathrm{U}_{w(z)} v\right)^{\prime},-i\left|\left(\mathrm{U}_{w(z)} v\right)_{n}\right|\right)=: V .
$$

Observe that $r_{\Omega}(z ; v)=r_{\Omega}\left(z ; e^{i \theta_{v}} v\right)$. In view of (3.2) and (3.3), we may focus on those $V$-writing $V=\left(V^{\prime},-i V_{n}\right)$ - such that $V^{\prime} \neq 0$ and $V_{n}>0$. We view a portion of $\partial \Omega$ around $w(z)$ after the application of the C-affine transformation $\mathbf{U}^{w(z)}$. It then follows from elementary coordinate geometry that if $\rho$ is a positive number that satisfies (recall that $z$ is mapped to $\left(0, \ldots, 0, i \cdot d_{\Omega}(z)\right)$ under $\left.\mathrm{U}^{w(z)}\right)$

$$
C \Psi_{\alpha}(\rho)+\rho \frac{V_{n}}{\left\|V^{\prime}\right\|}=d_{\Omega}(z)
$$

then the set $\left(0, \ldots, 0, i \cdot d_{\Omega}(z)\right)+\overline{D\left(0 ; \rho_{*}\right)} V$ intersects $\mathbf{M}$, where

$$
\rho_{*}:=\rho /\left\|V^{\prime}\right\| \text {. }
$$


Since

$$
\mathbf{M} \subset B^{n-1}\left(0 ; R_{0}\right) \times\left(\left(-s_{0}, s_{0}\right)+i\left(-s_{0}, s_{0}\right)\right) \backslash \mathrm{U}^{w(z)}(\Omega),
$$

it easily follows that $\left(0, \ldots, 0, i \cdot d_{\Omega}(z)\right)+\overline{D\left(0 ; \rho_{*}\right)} V$ intersects $\mathrm{U}^{w(z)}(\partial \Omega)$. Hence

$$
r_{\Omega}(z ; v)=r_{\mathrm{U}^{w(z)}(\Omega)}\left(\left(0, \ldots, 0, i \cdot d_{\Omega}(z)\right) ; V\right) \leq \rho_{*} .
$$

By (3.4) and the fact that $\Psi_{\alpha}$ is increasing on $(0, \infty)$ we get that $\rho \leq\left[\log \left(C / d_{\Omega}(z)\right)\right]^{-1 / \alpha}$. Therefore,

$$
\rho_{*} \leq\left[\log \left(\frac{C}{d_{\Omega}(z)}\right)\right]^{-1 / \alpha}+d_{\Omega}(z) \leq 2\left[\log \left(\frac{C}{d_{\Omega}(z)}\right)\right]^{-1 / \alpha} .
$$

The second inequality follows from the fact that $d_{\Omega}(z)<\min \left(s_{0}, x_{0}\right)$, where $x_{0}$ is defined by (3.1). The above inequality, together with (3.5) and the estimates in the first paragraph of this proof, gives the desired conclusion.

A key requirement of our proof is to transcribe an estimate for $\left\|f^{\prime}(\zeta)\right\|$ (here $f: \mathbf{D} \rightarrow \Omega$ is a complex geodesic) given in terms of $\kappa_{\Omega}\left(f(\zeta) ; f^{\prime}(\zeta)\right)$-which is provided by Graham's result - into an estimate given in terms of $\zeta \in \mathbf{D}$. One such tool is an estimate by Lempert [11, Proposition 12]. However, since our domains of interest are not strongly convex, we will need an extension of this estimate. This has been provided by Mercer, and is as follows:

Result 3.3. (Mercer, [12]) Let $\Omega$ be a bounded convex domain in $\mathbf{C}^{n}$ and let $f: \mathbf{D} \rightarrow \Omega$ be a complex geodesic. There exists a constant $\beta>1$ and constants $C_{1}, C_{2}>0$ such that

$$
C_{1}(1-|\zeta|) \leq d_{\Omega}(f(\zeta)) \leq C_{2}(1-|\zeta|)^{1 / \beta} \quad \forall \zeta \in \mathbf{D} .
$$

We are now in a position to give

The proof of Theorem 1.4. Let $f: \mathbf{D} \rightarrow \Omega$ be a complex geodesic. It is easy to argue that $f$ is a proper map: see [9, Proposition 4.6.3], for instance. Let $K$ be the compact set given by Lemma 3.2. By the properness of $f$, there exists a constant $r_{0}>0$ such that $f(\zeta) \in \Omega \backslash K$ whenever $1-r_{0}<|\zeta|<1$. By Result 3.1

$$
\left\|f^{\prime}(\zeta)\right\| \leq 2 r_{\Omega}\left(f(\zeta) ; f^{\prime}(\zeta)\right) \kappa_{\Omega}\left(f(\zeta) ; f^{\prime}(\zeta)\right)=\frac{2 r_{\Omega}\left(f(\zeta) ; f^{\prime}(\zeta)\right)}{1-|\zeta|^{2}} \quad \forall \zeta \in \mathbf{D}
$$

The equality between the second and the third expression is due to the fact that $f$ is a complex geodesic. For $\zeta \in \mathbf{D}$ such that $1-r_{0}<|\zeta|<1$, it follows from Lemma 3.2 and the above inequality that

$$
\left\|f^{\prime}(\zeta)\right\| \leq 4\left[\log \left(\frac{C}{d_{\Omega}(f(\zeta))}\right)\right]^{-1 / \alpha} \frac{1}{1-|\zeta|^{2}} \leq 4\left[\log \left(\frac{C}{d_{\Omega}(f(\zeta))}\right)\right]^{-1 / \alpha} \frac{1}{1-|\zeta|}
$$

Finally, we invoke Mercer's estimate, Result 3.3, to get

$$
\begin{aligned}
\left\|f^{\prime}(\zeta)\right\| & \leq 4\left[\log \left(\frac{C / C_{2}}{(1-|\zeta|)^{1 / \beta}}\right)\right]^{-1 / \alpha} \frac{1}{1-|\zeta|} \\
& \equiv K_{1}\left[\log \left(\frac{K_{2}}{1-|\zeta|}\right)\right]^{-1 / \alpha} \frac{1}{1-|\zeta|} \quad \forall \zeta: 1-r_{0}<|\zeta|<1
\end{aligned}
$$


where $K_{1}$ and $K_{2}$ are appropriate positive constants. Define a function $\Phi:\left[0, r_{0}\right) \rightarrow$ $[0,+\infty]$ as follows:

$$
\Phi(x):= \begin{cases}\frac{K_{1}}{x}\left[\log \left(\frac{K_{2}}{x}\right)\right]^{-1 / \alpha}, & \text { if } 0<x<r_{0} \\ +\infty, & \text { if } x=0 .\end{cases}
$$

Write $f=\left(f_{1}, \ldots, f_{n}\right)$. By (3.6), each component satisfies

$$
\left|f_{j}^{\prime}(\zeta)\right| \leq \Phi(1-|\zeta|) \quad \forall \zeta: 1-r_{0}<|\zeta|<1, j=1, \ldots, n .
$$

Given Proposition 2.1, the theorem will follow if we can show that the above $\Phi$ is of class $\mathbf{L}^{1}\left(\left[0, r_{0}\right)\right)$. The convergence of the integral of $\Phi$ is a standard example; it converges precisely when $0<\alpha<1$. Thus, by our assumption on $\alpha, f$ extends continuously up to $\partial \mathbf{D}$.

\section{References}

[1] Catlin, D.: Boundary invariants of pseudoconvex domains. - Ann. of Math. (2) 120:3, 1984, $529-586$.

[2] DeVore, R. A., and G. G. Lorentz: Constructive approximation. - Grundlehren Math. Wiss. 303, Springer-Verlag, Berlin, 1993.

[3] Dyakonov, K. M.: Equivalent norms on Lipschitz-type spaces of holomorphic functions. Acta Math. 178:2, 1997, 143-167.

[4] Fornæss, J. E., and L. LEE: Asymptotic behavior of Kobayashi metric in the normal direction. - Math. Z. 261:2, 2009, 399-408.

[5] Fornæss, J. E., L. LeE, and Y. Zhang: On supnorm estimates for $\bar{\partial}$ on infinite type convex domains in $\mathbf{C}^{2}$. - J. Geom. Anal. 21:3, 2011, 495-512.

[6] Halfpap, J., A. Nagel, and S. Wainger: The Bergman and Szegó kernels near points of infinite type. - Pacific J. Math. 246:1, 2010, 75-128.

[7] Garnett, J. B.: Bounded analytic functions. Revised first edition. - Springer, New York, NY, 2007.

[8] Graham, I.: Distortion theorems for holomorphic maps between convex domains in $\mathbf{C}^{n}$. Complex Variables Theory Appl. 15:1, 1990, 37-42.

[9] Kobayashi, S.: Hyperbolic complex spaces. - Grundlehren Math. Wiss. 318, Springer-Verlag, Berlin, 1998.

[10] Kuusi, T., and G. Mingione: Universal potential estimates. - J. Funct. Anal. 262:10, 2012, 4205-4269.

[11] Lempert, L.: La métrique de Kobayashi et la représentation des domaines sur la boule. - Bull. Soc. Math. France 109, 1981, 427-474.

[12] Mercer, P. R.: Complex geodesics and iterates of holomorphic maps on convex domains in $\mathbf{C}^{n}$. - Trans. Amer. Math. Soc. 338:1, 1993, 201-211.

[13] Royden, H., and P. M. Wong: Carathéodory and Kobayashi metrics on convex domains. Unpublished manuscript, 1983.

[14] Zimmer, A. M.: Characterizing polynomial domains by their automorphism group. - Preprint, arXiv:1506.07852. 